\title{
Biophysical model of odor representation and processing in the rat olfactory bulb
}

\author{
Aditya Gilra, Upinder S Bhalla* \\ From Nineteenth Annual Computational Neuroscience Meeting: CNS*2010 \\ San Antonio, TX, USA. 24-30 July 2010
}

Experimental findings from our lab [1] indicate that mitral cells in the rat olfactory bulb respond to odor mixtures by weighted summation of odor components. Thus, responses to odor mixtures, morphing from odor A to odor B, can be fitted as weighted sums of responses to individual components A and B. Over $70 \%$ of odor morphs show a gradual shift in response, thus ruling out attractor dynamics, which would predict sharp transitions. Ongoing experiments indicate that summation in time is also linear at fixed concentration. Thus the response to any on-off odor pulse sequence can be predicted as a convolution of this stimulus sequence with the response to a narrow odor pulse (impulse response). We are therefore interested in analyzing how detailed physiological models of olfactory bulb neurons and their network interactions could give rise to this surprisingly simple computational behavior. This is especially interesting given the complexity and nonlinearity of the circuit and neurons in the olfactory bulb.

Modeling and simulation: Here we present a biophysically realistic model of the network of mitral and granule cells in the rat olfactory bulb. Simulations of this model replicate the weighted-sum-sigmoid responses of mitral cells to odor mixtures. Over the odor concentration ranges where summation is linear, we find that the convolution property of linear systems is applicable. Sister mitral cells (efferent from the same glomerulus) receive different lateral connections from other mitral cells via granule cells. We show that this causes them to respond differently, in their phase with respect to respiratory cycle, making them non-redundant, as observed in recent in-review experimental findings.

Representation and processing: We consider the activity of $\mathrm{N}$ glomeruli, as an $\mathrm{N}$-dimensional input odor

\footnotetext{
* Correspondence: bhalla@ncbs.res.in

National Centre for Biological Sciences, Tata Institute of Fundamental

Research, Bellary Road, Bangalore, 560065, India
}

space to the olfactory bulb. Each mitral cell, by means of weighted-summation, acts to project the input odor vector onto a different 'direction' in the odor space, defined by its connectivity. This representation is further enhanced by the different respiration tuning of each mitral cell to different odors. We propose that subsequent processing layer(s), perhaps in the olfactory cortex may use combinations of this panoply of projections and respiratory tuning to extract features or identify principal components.

Robustness and Decorrelation: We analyze the robustness of odor representations in the olfactory bulb with respect to system noise and loss / change of connectivity / neurons. We further study decorrelation in the output vis-a-vis the input.

\section{Conclusion}

Our biophysical simulations suggest that the mitral cell temporal response is shaped independently by the primary and secondary dendritic inputs. This allows a weighted-sum response to a mixture of odors which is even linear in time over appropriate concentration ranges. We show that this representation of odors by the olfactory bulb is conducive to feature extraction and robust against noise and degradation.

\section{Published: 20 July 2010}

\section{Reference}

1. Adil G. Khan, Mukund Thattai, Upinder S. Bhalla: Odor representations in the rat olfactory bulb change smoothly with morphing stimuli. Neuron 2008, 57(4):571-585.

doi:10.1186/1471-2202-11-S1-P63

Cite this article as: Gilra and Bhalla: Biophysical model of odor representation and processing in the rat olfactory bulb. BMC Neuroscience 2010 11(Suppl 1):P63. 